\title{
5-Fluorouracil Encapsulated Chitosan Nanoparticles for pH-Stimulated Drug Delivery: Evaluation of Controlled Release Kinetics
}

\author{
R. Seda Tığlı Aydın ${ }^{1,2}$ and Mehlika Pulat ${ }^{2}$ \\ ${ }^{1}$ Biomedical Engineering Department, Zonguldak Karaelmas University, 67100 Zonguldak, Turkey \\ ${ }^{2}$ Chemistry Department, Gazi University, Beşevler, 06500 Ankara, Turkey
}

Correspondence should be addressed to R. Seda Tiğlı Aydın, rseda.tigli@gmail.com

Received 9 December 2011; Accepted 30 March 2012

Academic Editor: Jinquan Wei

Copyright (c) 2012 R. S. Tığlı Aydın and M. Pulat. This is an open access article distributed under the Creative Commons Attribution License, which permits unrestricted use, distribution, and reproduction in any medium, provided the original work is properly cited.

\begin{abstract}
Nanoparticles consisting of human therapeutic drugs are suggested as a promising strategy for targeted and localized drug delivery to tumor cells. In this study, 5-fluorouracil (5-FU) encapsulated chitosan nanoparticles were prepared in order to investigate potentials of localized drug delivery for tumor environment due to $\mathrm{pH}$ sensitivity of chitosan nanoparticles. Optimization of chitosan and 5-FU encapsulated nanoparticles production revealed $148.8 \pm 1.1 \mathrm{~nm}$ and $243.1 \pm 17.9 \mathrm{~nm}$ particle size diameters with narrow size distributions, which are confirmed by scanning electron microscope (SEM) images. The challenge was to investigate drug delivery of 5-FU encapsulated chitosan nanoparticles due to varied $\mathrm{pH}$ changes. To achieve this objective, $\mathrm{pH}$ sensitivity of prepared chitosan nanoparticle was evaluated and results showed a significant swelling response for $\mathrm{pH} 5$ with particle diameter of $\sim 450 \mathrm{~nm}$. In vitro release studies indicated a controlled and sustained release of 5-FU from chitosan nanoparticles with the release amounts of $29.1-60.8 \%$ due to varied $\mathrm{pH}$ environments after $408 \mathrm{~h}$ of the incubation period. $\mathrm{pH}$ sensitivity is confirmed by mathematical modeling of release kinetics since chitosan nanoparticles showed stimuli-induced release. Results suggested that 5-FU encapsulated chitosan nanoparticles can be launched as $\mathrm{pH}$-responsive smart drug delivery agents for possible applications of cancer treatments.
\end{abstract}

\section{Introduction}

The potential use of nanoparticles as drug carriers has been represented over the last few years as an important challenge, since nanoparticles have been designed to improve the pharmacological and therapeutic effects in terms of reducing their toxic side effects $[1,2]$. Nanoparticles as drug carriers not only allow the continuous and controlled release of therapeutic drugs to maintain drug levels within a desired level but also include localizing and specifically targeting the drugs to their intended tissues and cells, thereby decreasing drug doses and improving patient compliance [3].

Recently, chitosan has attracted a great attention in pharmaceutical and biomedical fields because of its advantageous biological properties, such as biodegradability, biocompatibility, and nontoxicity $[4,5]$. Chitosan is a cationic polysaccharide which is obtained by partial deacetylation of chitin.
In contrast to other polymers, chitosan is a hydrophilic polymer with positive charge, which reveals a special characteristic to chitosan from the technological point of view [6]. The process for production of chitosan nanoparticles depends on the approach on ionic gelation, where nanoparticles are formed by means of electrostatic inter actions between the positively charged chitosan chains and polyanions employed as cross-linkers like tripolyphosphate (TPP). Tripolyphosphate (TPP) has been popular because it is nontoxic, has the ability to gel quickly, and interacts electrostatically with cationic chitosan. Recently, there has been a substantial interest on chitosan nanoparticles since production process is simple and mild. Chitosan nanoparticles, which are launched as promising carriers for controlled-release drug delivery, are exploited extensively in the pharmaceutical industry [7-10].

Stimuli response is an important property that affects the ability of systems to deliver drugs effectively. Drug release 
could be triggered by physical or chemical stimuli, such as $\mathrm{pH}$, ionic strength, temperature, and magnetic and biological molecules. Thus, stimuli-responsive nanoparticles might be a promise carrier system since the particles can selectively respond to environmental change in vitro or in vivo [11]. Chitosan exhibits a $\mathrm{pH}$-sensitive behavior due to the large quantities of amino groups on its chains [12]. Due to $\mathrm{pH}$ sensitivity, chitosan nanoparticles, introduced as chitosan nanogels, can undergo volume phase transitions from swollen to collapsed states or vise versa. This feature is extremely important from the applicability point of view, since the delivery and release capacity of the particles significantly changes from a swollen to a collapsed state, which renders chitosan nanoparticles promising carriers for the delivery of macromolecules [13].

5-Fluorouracil (5-FU) has been used for the treatment of solid tumors, which employed most extensively in clinical chemotherapy for the treatment of carcinomas of the colon or rectum and also precancerous dermatoses. However, like other drugs used for chemotherapy, it affects the growth of normal body cells and often causes side effects such as hair loss, fatigue, birth defects, mouth sores and ulcers, liver disease, and a temporary drop in bone marrow function [14, 15]. Thus, it has been suggested that chitosan nanoparticles might prevent the side effects induced by 5-FU [8]. Yang and colleagues investigated 5-FU-loaded chitosan nanoparticles whether they could be used as effective drug delivery systems and suggested that chitosan-drug conjugates are expected to overcome the problematic side effects, which were induced by $5-\mathrm{FU}$, of low-molecular-weight prodrugs [8]. However, $\mathrm{pH}$ sensitivity of chitosan nanoparticles with regard to 5-FU release behavior and further mathematical modeling on release kinetics remains unproven although it is well known that a certain kind of abnormal tissues has only slightly more acidic environment than normal tissue [16].

In tumor tissues, a $\mathrm{pH}$ value of 6 is easily accomplished because of the lactic acid produced as a byproduct of anaerobic glucose metabolism. Even lower $\mathrm{pH}$ values of 3.0-5.5 are feasible in acidic intracellular organelles, such as endosomes and lysosomes, within cancer cells [17]. In the present study, we prepared TPP-crosslinked 5-FU encapsulated chitosan nanoparticles and demonstrated optimum conditions of production for tumor-localized drug delivery applications. $\mathrm{pH}$ sensitivies of chitosan nanoparticles were investigated, and the release of 5-FU from chitosan nanoparticles were evaluated by further mathematical models whether they could be used as drug delivery agents for cancer treatments.

\section{Materials and Methods}

Chitosan, derived from crab shell, in the form of flakes was purchased from Sigma-Aldrich (Medium Molecular Weight, Catalogue no. 448877). The degree of deacetylation and molecular weight for the medium-molecular-weight chitosan (MWM chitosan) is $75-85 \%$ and $190-310 \mathrm{kDa}$ based on viscosity, respectively. Sodium tripolyphosphate (TPP) (purity: 85\%), 5-fluorouracil (5-FU) (purity: 99\%), and phosphate-buffered saline (PBS) tablets ( $\mathrm{pH} 7.4$ ), were purchased from Sigma-Aldrich Chemical Co. Ltd. All other reagents were in analytical grade.

2.1. Preparation of Chitosan Nanoparticles. Chitosan nanoparticles were produced based on ionic gelation of TPP with chitosan after chitosan purification as described elsewhere [10]. Chitosan was dissolved in $1 \%(\mathrm{v} / \mathrm{v})$ acetic acid solution to make up chitosan concentrations at $0.50,0.75,1.00,1.25$, 2.50 , and $5.00(\mathrm{mg} / \mathrm{mL})$. Tween 80 (Sigma, Germany) $(0.5 \%$ $(\mathrm{v} / \mathrm{v}))$, as a resuspending agent, was added to chitosan solutions in order to prevent particle aggregation, and then chitosan solutions were raised to $\mathrm{pH} 4.6-4.8$ with $1 \mathrm{~N} \mathrm{NaOH}$. TPP was dissolved in distilled water to maintain TPP solutions of $0.50,0.75,1.00,1.25,2.50$, and $5.00(\mathrm{mg} / \mathrm{mL})$. All solutions were filtered through 0.22 micron filter (Millipore). Prepared chitosan solutions were flushed mixed with TPP solutions with a volumetric ratio of $2.5: 1(\mathrm{v} / \mathrm{v})$ (chitosan:TPP) under magnetic stirring at room temperature. The formation of chitosan-TPP nanoparticles started spontaneously via the TPP-initiated ionic gelation mechanism. Nanoparticles were purified by centrifugation at $12000 \mathrm{~g}$ for $30 \mathrm{~min}$. Supernatants were discarded and resuspended in water, and the chitosan nanoparticles were then freeze-dried before further use or analysis.

2.2. Dynamic Light Scattering (DLS) Analysis. Particle size distribution of chitosan nanoparticles was analyzed through DLS with Zetasizer Nano S (Malvern, UK). The analysis was performed in triplicate at a temperature of $25^{\circ} \mathrm{C}$.

\subsection{Evaluation of Optimum Criteria for Chitosan Nanoparticle Preparation. In order to evaluate effective factors during chitosan nanoparticle production, nanoparticles with the smallest and the highest particle sizes, determined from DLS analysis, were chosen as two models for further optimization studies. At first, chosen chitosan nanoparticles were repro- duced by changing chitosan:TPP ratios as $1: 1,1.5: 1,2: 1$, $2.5: 1,3: 1$, and $3.5: 1(\mathrm{v} / \mathrm{v})$. Then, the effect of Tween 80 was investigated by changing the amounts as $0.0 \%, 0.5 \%$, and $1.0 \%$. Finally, all chitosan nanoparticles were investigated in terms of particle size, and optimum conditions were evaluated by selection of optimum criteria for chitosan nano- particle production.}

\subsection{Investigation of $p H$ Sensitivity of Chitosan Nanoparticles.} Chitosan nanoparticles were soaked in phosphate buffer solutions (PBSs) at room temperature with different $\mathrm{pH}$ values of $3,4,5,6$, and 7.4 in order to investigate $\mathrm{pH}$ responsive behavior of nanoparticles. $\mathrm{pH}$ of prepared PBS solutions was arranged by adding $\mathrm{HCl}(37 \%)$ and $\mathrm{NaOH}(50(\mathrm{w} / \mathrm{v}) \%)$ solutions. Chitosan nanoparticles were incubated for 1 and $3 \mathrm{~h}$ ensuring for swelling equilibrium. Data of particle size distribution obtained in different $\mathrm{pH}$ solution after 1 and $3 \mathrm{~h}$ incubation period was measured by Zetasizer Nano S (Malvern, UK).

2.5. Preparation of 5-FU Encapsulated Chitosan Nanoparticles. Chitosan was dissolved in $1 \%(\mathrm{v} / \mathrm{v})$ acetic acid solution to 
maintain chitosan concentration at $0.75(\mathrm{mg} / \mathrm{mL})$. Prepared chitosan solutions were mixed with 5 -FU solutions (5-FU dissolved in water), and $0.5,1.0$, and $5 \mathrm{mg} / \mathrm{mL} 5$-FU containing chitosan solutions were maintained. Tween 80 (Sigma, Germany) $(0.5 \%(\mathrm{v} / \mathrm{v}))$ was added to chitosan solutions, and $\mathrm{pH}$ was arranged as 4.6-4.8. Prepared 5-FU-containing chitosan solutions were flushed mixed with $0.5 \mathrm{mg} / \mathrm{mL}$ TPP solutions with a ratio of volume ratio of $(2: 1)(\mathrm{v} / \mathrm{v})$ (chitosan:TPP). The nanoparticle suspension was gently stirred for $20 \mathrm{~min}$ at room temperature to allow excess 5-FU adsorption on the nanoparticles to reach isothermal equilibrium. 5-FU encapsulated chitosan nanoparticles were centrifuged at $12000 \mathrm{~g}$ for $30 \mathrm{~min}$, resuspended in water and freeze-dried used for further analyses. Prepared 5-FU encapsulated chitosan nanoparticles were analyzed by Zetasizer Nano S (Malvern,UK) in order to determine mean average particle size distributions.

2.6. Scanning Electron Microscopy (SEM). The morphological characteristics of both chitosan and 5-FU encapsulated chitosan nanoparticles were examined by scanning electron microscope (SEM) (FEI, Nova 600 Nano SEM). One drop of dilute chitosan nanoparticles' solution was dropped on a carbon film and let air-dried before viewing.

2.7. Fourier Transform Infrared (FTIR) Spectra Studies. FTIR spectra of chitosan and 5-FU encapsulated chitosan nanoparticles were recorded on $\mathrm{KBr}$ pellets with a FT-IR spectrophotometer (Thermo Scientific Nicolet iS10, USA)

2.8. Evaluation of 5-FU Encapsulation. Encapsulation efficiencies of prepared chitosan nanoparticles were determined by indirect method. Briefly, nanoparticles were centrifuged at $12,000 \mathrm{~g}$ at $30 \mathrm{~min}$, and then supernatants of 5 -FU solutions were measured by UV-spectrophotometer (Hitachi 150-20, Japan) at $266 \mathrm{~nm}$. Calculations were performed by using the calibration curve, and encapsulation efficiencies were calculated as follows:

$$
\begin{aligned}
5-\mathrm{FU} \text { encapsulation efficiency }(\%)= & \frac{\text { total } 5-\mathrm{FU}-\text { free } 5-\mathrm{FU}}{\text { total } 5-\mathrm{FU}} \\
& \times 100 .
\end{aligned}
$$

2.9. 5-FU Release Studies. 5-FU release studies from chitosan nanoparticles were performed in prepared PBS solutions with various $\mathrm{pH}$ values $(3,4,5,6$, and 7.4). Chitosan nanoparticles, prepared with known initial 5-FU concentrations $(0.5 \mathrm{mg} / \mathrm{mL} 5-\mathrm{FU})$, were suspended in $1 \mathrm{~mL}$ buffered solutions and then transferred into tubular cellulose membranes (Sigma, Germany). Chitosan nanoparticles in cellulose membranes were then incubated in $10 \mathrm{~mL}$ different $\mathrm{pH}$-buffered reservoirs at $37^{\circ} \mathrm{C}$ with $35 \mathrm{rpm}$ agitation. The amount of 5-FU released was determined from the nanoparticles at selected time intervals through spectrophotometric investigation with measurement at $266 \mathrm{~nm}$.
2.10. Mathematical Modeling and Release Kinetics. In order to investigate the mechanism of 5-FU release from chitosan nanoparticles, in vitro release data were analyzed using various kinetic models. The zero-order kinetic model (2) describes the release from the system where the release rate is independent of its concentration of the dissolved substance [18]:

$$
Q_{t}=Q_{0}+K_{0} t
$$

where $Q_{0}=$ initial amount of drug, $Q_{t}=$ cumulative amount of drug release at time " $t$ ", $K_{0}=$ zero-order release constant.The first-order release (3) defines where the release rate is concentration dependent [19]:

$$
\log Q_{t}=\log Q_{0}+K_{1} t
$$

where $K_{1}$ is the first-order release constant.

Higuchi described the release of drugs from insoluble matrix as a square root of time-dependent process based on the Fickian diffusion [20]:

$$
Q_{t}=Q_{0}+K_{\mathrm{H}} t^{1 / 2},
$$

where $K_{\mathrm{H}}$ is the Higuchi constant.

The Hixson-Crowell cube root law (5) defines the release from systems by dissolution where there is a change in surface area and diameter of particles [21]:

$$
\sqrt[3]{Q_{t}}-\sqrt[3]{Q_{0}}=K_{\mathrm{HC}} t
$$

where $K_{\mathrm{HC}}$ is the Hixson-Crowell constant.

Korsmeyer et al. derived a simple relationship, the Korsmeyer-Peppas model, which described drug release from a polymeric system [22]:

$$
Q_{t}=K_{\mathrm{KP}} t^{n},
$$

where $K_{\mathrm{KP}}$ is the Korsmeyer-Peppas constant and $n$ is the release exponent describing the drug release mechanism.

The Kopcha model (7) describes how to quantify the contribution of diffusion and polymer relaxation [23]:

$$
Q_{t}=A t^{1 / 2}+B t,
$$

where $A$ and $B$ are the Kopcha constants.

Modeling was performed using the parameters that provide the closest fit between experimental observations and the nonlinear function. The model that best fits the release data was selected based on the correlation coefficient $\left(R^{2}\right)$ in models described above. The model, which gives highest $R^{2}$ value, is considered as the best fit of release data.

\section{Results and Discussion}

In the present study, we focused on chitosan nanogels, nanoparticles which have smart features for drug delivery concerning cancer treatments. Description of such kind of system should have some important properties: (i) obtained spontaneously under exceptionally mild conditions without 
TABLE 1: Average particle size of chitosan nanoparticles prepared at different concentrations. ${ }^{1}$

\begin{tabular}{|c|c|c|c|c|}
\hline $\begin{array}{l}\text { Chitosan } \\
(\mathrm{mg} / \mathrm{mL}) \\
\end{array}$ & $\begin{array}{c}\mathrm{TPP} \\
(\mathrm{mg} / \mathrm{mL})\end{array}$ & $\begin{array}{c}\text { Average particle size } \\
(\mathrm{nm})\end{array}$ & Visual identification & $\begin{array}{l}\text { Polydispersity index } \\
\text { (PDI) }\end{array}$ \\
\hline 0.50 & 0.50 & $190.8 \pm 20.5$ & Opalescent solution & 0.094 \\
\hline 0.50 & 0.75 & $>1000$ & Aggregates & $*$ \\
\hline 0.50 & 1.0 & $>1000$ & Aggregates & $*$ \\
\hline 0.50 & 1.25 & $>1000$ & Aggregates & $*$ \\
\hline 0.75 & 0.50 & $152.6 \pm 3.3$ & Opalescent solution & 0.232 \\
\hline 0.75 & 0.75 & $228.2 \pm 2.9$ & Opalescent solution & 0.080 \\
\hline 0.75 & 1.0 & $>1000$ & Aggregates & $*$ \\
\hline 0.75 & 1.25 & $>1000$ & Aggregates & $*$ \\
\hline 1.0 & 0.50 & $181.7 \pm 2.1$ & Opalescent solution & 0.347 \\
\hline 1.0 & 0.75 & $195.4 \pm 3.5$ & Opalescent solution & 0.333 \\
\hline 1.0 & 1.0 & $251.9 \pm 3.5$ & Opalescent solution & 0.259 \\
\hline 1.0 & 1.25 & $>1000$ & Aggregates & $*$ \\
\hline 1.25 & 0.50 & $195.2 \pm 3.1$ & Opalescent solution & 0.388 \\
\hline 1.25 & 0.75 & $244.5 \pm 8.9$ & Opalescent solution & 0.377 \\
\hline 1.25 & 1.0 & $286.5 \pm 3.8$ & Opalescent solution & 0.056 \\
\hline 1.25 & 1.25 & $393.1 \pm 1.8$ & Opalescent solution & 0.443 \\
\hline 1.25 & 2.5 & $>1000$ & Opalescent solution & $*$ \\
\hline 1.25 & 5.0 & $>1000$ & Opalescent solution & $*$ \\
\hline 2.50 & 1.25 & $924.9 \pm 40.1$ & Aggregates & 0.423 \\
\hline 2.50 & 2.50 & $>1000$ & Aggregates & $*$ \\
\hline 2.50 & 5.0 & $>1000$ & Aggregates & $*$ \\
\hline 5.0 & 1.25 & $>1000$ & Aggregates & * \\
\hline 5.0 & 2.50 & $>1000$ & Aggregates & $*$ \\
\hline 5.0 & 5.0 & $>1000$ & Aggregates & $*$ \\
\hline
\end{tabular}

${ }^{1}$ Chitosan : TPP $(2.5: 1)(\mathrm{v} / \mathrm{v})$ Tween $80 ; 0.5 \%(\mathrm{v} / \mathrm{v})$; measurements are performed three times.

$*$ PDI $>1.00$.

involving high temperatures and organic solvents, (ii) has a valuable drug loading capacity and provides a continuous and sustainable release of the encapsulated drug for several days, and (iii) has a $\mathrm{pH}$-sensitive behavior. The properties basically concern optimizing general conditions of chitosan nanoparticle production and the feasibility of drug entrapment and release with regard to cancer treatment applications. In terms of appropriate localized drug delivery by means of tumor treatment, chitosan and 5-FU encapsulated chitosan nanoparticles were produced, due to the certain properties mentioned above.

3.1. Chitosan Nanoparticle Production Conditions. Chitosan's ability of quick gelling on contact with polyanions relies on the formation of inter- and intramolecular crosslinkages mediated by polyanions [24]. The preparation of chitosan nanoparticles is based on an ionic gelation interaction between positively charged chitosan and negatively charged tripolyphosphate (TPP) at room temperature immediately [25, 26]. TPP is a multivalent anion that possesses negative charges; chitosan in acidic solution has amino groups that can undergo protonation. During the preparation process, TPP electrostatically attracted to the $\mathrm{NH}_{3}{ }^{+}$groups in chitosan to produce ionically crosslinked chitosan nanoparticles
$[8,27]$. Size and size distribution of the chitosan nanoparticles depend largely on concentration of chitosan and TPP solutions. For the success of chitosan with nanosized scale, the concentration of chitosan and TPP should be controlled at a suitable range [28].

The mean size and size distribution of each batch of chitosan nanoparticle suspension were analyzed using the Zetasizer analysis. Table 1 represents effects of chitosan and TPP concentrations on particle size and ability to produce nanoparticle by visual observations. Previously it had been shown that the appearance of the solution changed when a certain amount of TPP ions was added to the chitosan solution, from a clear to opalescent solution that indicated a change of the physical states of the chitosan to form nanoparticles, then microparticles, and eventually aggregates [29]. In this study, samples were visually analyzed and identified as clear solution, opalescent suspension, and aggregates (Table 1). As seen from Table 1, chitosan concentration was highly effective in nanoparticle production, and minimum criteria for nanoparticle formation should be lower than $2.5 \mathrm{mg} / \mathrm{mL}$ chitosan concentration for selected TPP concentrations. However, below $2.5 \mathrm{mg} / \mathrm{mL}$ chitosan concentration, when the TPP concentration exceeds chitosan concentration, the chitosan aggregation was formed, which might be due to 
TABLE 2: Average particle size of chitosan nanoparticles prepared at different chitosan/TPP ratios. ${ }^{1}$

\begin{tabular}{lcc}
\hline $\begin{array}{l}\text { Chitosan: TPP } \\
\text { ratio (v/v) }\end{array}$ & $\begin{array}{c}1.25 \mathrm{mg} / \mathrm{mL} \\
\text { chitosan }: 1.25 \mathrm{mg} / \mathrm{mL} \\
\text { TPP }\end{array}$ & $\begin{array}{c}0.75 \mathrm{mg} / \mathrm{mL} \\
\text { chitosan }: 0.5 \mathrm{mg} / \mathrm{mL} \\
\text { TPP }\end{array}$ \\
\hline $1: 1$ & $>1000^{*}$ & $>1000^{*}$ \\
$2: 1$ & $>1000^{*}$ & $148.8 \pm 1.1$ (PDI: 0.066$)$ \\
$2.5: 1$ & $393.1 \pm 1.8$ (PDI: 0.443$)$ & $152.6 \pm 3.3$ (PDI: 0.232$)$ \\
$3: 1$ & $421.9 \pm 27.6$ (PDI: 0.377$)$ & $162.3 \pm 7.8$ (PDI: 0.367 ) \\
$3.5: 1$ & $510.0 \pm 42.9$ (PDI: 0.379$)$ & $178.1 \pm 2.9$ (PDI: 0.295$)$ \\
\hline
\end{tabular}

${ }^{1}$ Tween $800.5 \%(\mathrm{v} / \mathrm{v})$; measurements are performed three times.

$*$ PDI $>1.00$.

the fact that more chitosan chains were crosslinked in the presence of a high concentration of TPP. In the range of minimum criteria for nanoparticle formation (chitosan concentration $<2.5 \mathrm{mg} / \mathrm{mL}$ and chitosan concentration should not exceed TPP concentration), at fixed chitosan concentration, mean diameter of nanoparticles increases with the elevation of TPP concentration. Results showed that minimum and maximum mean size diameters of prepared chitosan nanoparticles were $152.6 \pm 3.3 \mathrm{~nm}$ and $393.1 \pm 1.8 \mathrm{~nm}$, respectively. The size distribution profiles represent minimum and maximum average size diameters with narrow size distributions (polydispersity index $<1$ ).

Previously it has been shown that chitosan-to-TPP weight ratio prominently increases nanoparticle size [24]. Table 2 presents effects of chitosan to TPP volume ratios on selected chitosan nanoparticles (minimum and maximum mean size diameter) from Table 1 . Results showed that the increment of chitosan-to-TPP volume ratio effectively increase particle size diameter. Moreover, while minimum chitosan : TPP ratio is $2.5: 1$ for the selected maximum-sized nanoparticle $(1.25 \mathrm{mg} / \mathrm{mL}: 1.25 \mathrm{mg} / \mathrm{mL})$, the minimum chitosan : TPP ratio is determined as $2: 1$ for the selected minimum-sized nanoparticle $(0.75 \mathrm{mg} / \mathrm{mL}: 0.5 \mathrm{mg} / \mathrm{mL})$. Above these values, nanoparticle formation could not be achieved, and chitosan nanoparticle is produced with a minimum mean size diameter of $148.8 \pm 1.1 \mathrm{~nm}$.

Another parameter, which should be considered for successful chitosan nanoparticle formation, is the optimization of Tween 80 agent during nanoparticle formation. Table 3 presents effects of Tween 80 amount on chitosan nanoparticle formation. Results showed that Tween 80, suspending agent, must be employed on nanoparticle production process. During chitosan nanoparticle formation, using Tween 80 agent is critical, since chitosan nanoparticle formation in the tested region area could not be achieved without this agent. However, as seen from Table 3, elevated amount of Tween 80 increased particle size. The size distribution profiles of chitosan nanoparticles $(0.75 \mathrm{mg} / \mathrm{mL}: 0.5 \mathrm{mg} / \mathrm{mL})$ showed highly uniform $148.8 \pm 1.1 \mathrm{~nm}(\mathrm{PDI}=0.066)$ and $177.0 \pm 3.2 \mathrm{~nm}(\mathrm{PDI}=0.090)$ particle size diameters for $0.5 \%$ and $1.0 \%$ Tween 80 , respectively. Results concluded that $0.75 \mathrm{mg} / \mathrm{mL}$ chitosan and $0.5 \mathrm{mg} / \mathrm{mL}$ TPP solutions with $2: 1$ (chitosan: TPP) volume ratio in the presence of $0.5 \%$ Tween 80 could succeed in the formation of minimum particle size
TABLE 3: Average particle size of chitosan nanoparticles prepared at different amounts of Tween $80 .^{1}$

\begin{tabular}{lcc}
\hline $\begin{array}{l}\text { Tween } 80 \\
(\%)\end{array}$ & $\begin{array}{c}1.25 \mathrm{mg} / \mathrm{mL} \\
\text { chitosan }: 1.25 \mathrm{mg} / \mathrm{mL} \\
\text { TPP }(2.5: 1)(\mathrm{v} / \mathrm{v})\end{array}$ & $\begin{array}{c}0.75 \mathrm{mg} / \mathrm{mL} \\
\text { chitosan }: 0.5 \mathrm{mg} / \mathrm{mL} \\
\text { TPP }(2: 1)(\mathrm{v} / \mathrm{v})\end{array}$ \\
\hline 0 & $>1000^{*}$ & $>1000^{*}$ \\
0.5 & $393.1 \pm 1.8$ (PDI: 0.443$)$ & $148.8 \pm 1.1($ PDI: 0.066$)$ \\
1.0 & $432.8 \pm 10.0$ (PDI: 0.436$)$ & $177.0 \pm 3.2($ PDI: 0.090$)$ \\
\hline
\end{tabular}

${ }^{1}$ Measurements are performed three times.

$*$ PDI $>1.00$

diameter $(148.8 \pm 1.1 \mathrm{~nm})$ of chitosan nanoparticles. However, it had been shown that parameters like molecular weight and deacetylation degree of chitosan dominantly affect chitosan nanoparticle size $[24,28,30]$.

It is well known that particle size plays an important role on mucosal and epithelial tissue uptake of nanoparticles and on the alternation of pharmacokinetics by affecting the tissue distribution and clearance [4]. With regard to all these data, minimum mean diameter size chitosan nanoparticles $(148.8 \pm 1.1 \mathrm{~nm})$ were selected for further studies investigated in this paper.

3.2. $p H$ Sensitivity of Chitosan Nanoparticles. As the chitosan network contains $\mathrm{pH}$-ionizable groups, a $\mathrm{pH}$ variation will modify the network electrical state and thus the swelling behavior. In order to evaluate $\mathrm{pH}$ dependency of chitosan nanoparticles $(0.75 \mathrm{mg} / \mathrm{mL}$ chitosan, $0.5 \mathrm{mg} / \mathrm{mL}$ TPP, chitosan: TPP $(2: 1))$, particles were soaked in phosphate buffers with various $\mathrm{pH}$ values $(\mathrm{pH}: 3,4,5,6$, and 7.4) and mean size diameters of the particles were measured. Figure 1 presents average size diameters of chitosan nanoparticles via different $\mathrm{pH}$ values of phosphate buffer solutions after 1 and $3 \mathrm{~h}$ incubation period. As seen from Figure 1, particle size sharply increased when $\mathrm{pH}$ moved up from 3 to 5 . On the other hand, a considerable decrease of particle size was determined when $\mathrm{pH}$ exceeded 5 and shifted to 7.4 (Figure 1). This $\mathrm{pH}$-responsive behavior could be ascribed to the protonation of the primary amino group on the chitosan chain, resulting in the increase of electric density and repulsion force between crosslinked chitosan chains. Besides, Figure 1 demonstrates that there is no statistically significant difference on chitosan nanoparticle size between 1 and $3 \mathrm{~h}$ incubation period, indicating that chitosan nanoparticles reached equilibrium swelling in less than $1 \mathrm{~h}$ (equilibrium swelling time: $50 \mathrm{~min}$ ). Results concluded that particle size is very sensitive to the changing $\mathrm{pH}$ values of the residing aqueous environment, indicating that the surface density of protonised amino groups and the degree of protonisation are reversibly responsive to changing solution $\mathrm{pH}$ values. In this study, swelling and shrinking mechanism with regard to swinging $\mathrm{pH}$ values has been investigated in terms of smart responsive nanoparticle systems for localized drug delivery. Chitosan nanoparticles were demonstrated as $\mathrm{pH}$ sensitive with a reversible process of swelling and shrinking of particles, ranging from $\sim 450 \mathrm{~nm}$ to $\sim 150 \mathrm{~nm}$ of particle size diameter. In tumor cells, low $\mathrm{pH}$ values (around 6) 
TABLE 4: Average particle size of 5-FU encapsulated chitosan nanoparticles. ${ }^{1}$

\begin{tabular}{lcccc}
\hline Chitosan nanoparticle & $\begin{array}{c}\text { Average particle size } \\
(\mathrm{nm})\end{array}$ & $\begin{array}{c}\text { Polydispersity index } \\
(\mathrm{PDI})\end{array}$ & Visual identification & $\begin{array}{c}\text { Encapsulation } \\
\text { efficiency }(\%)\end{array}$ \\
\hline $0.5 \mathrm{mg} / \mathrm{mL} \mathrm{5-FU}$ & $243.1 \pm 17.9$ & 0.091 & Opalescent & $69.69 \pm 0.3$ \\
$1.0 \mathrm{mg} / \mathrm{mL} 5-\mathrm{FU}$ & $86.9 \pm 1.4$ & 0.262 & Clear solution & $31.23 \pm 0.9$ \\
$5.0 \mathrm{mg} / \mathrm{mL} 5-\mathrm{FU}$ & $69.1 \pm 1.0$ & 0.256 & Clear solution & $29.98 \pm 0.8$ \\
\hline
\end{tabular}

${ }^{1}$ Chitosan: TPP ratio $2: 1(\mathrm{v} / \mathrm{v})$ Tween $800.5 \%(\mathrm{v} / \mathrm{v})$; measurements are performed three times.

are accomplished due to the anaerobic glucose metabolism. Moreover, $\mathrm{pH}$ values of 3.0-5.5 are feasible in acidic intracellular organelles, such as endosomes and lysosomes, within cancer cells [17]. Thus, prepared chitosan nanoparticles would be expected to have an effective drug release in the tumor environment since the drug release rate can be suddenly accelerated with lower $\mathrm{pH}$ (around 5) after endocytosis into cancer cells.

3.3. 5-FU Encapsulated Chitosan Nanoparticles. 5-FU encapsulated chitosan nanoparticles were prepared by ionic crosslinking method between TPP and chitosan solutions having $0.5,1.0$, and $5.0 \mathrm{mg} / \mathrm{mL} 5-\mathrm{FU}$ concentrations. In Table 4, average particle size diameters of 5-FU encapsulated chitosan nanoparticles are presented. As seen from Table 4, nanoparticle size dramatically decreased with the increase of 5-FU concentration in chitosan solutions, which may be due to the simultaneous electrostatic interaction attractions between TPP and 5-FU with chitosan. 5-FU encapsulated chitosan nanoparticles formed instantaneously when polyanionic TPP is added to readily mixed chitosan-5-FU solutions. The formation of nanoparticles depends dramatically on the concentration of free amino groups, which strengthens the electrostatic interactions between the nanoparticles and the drug, helping to reduce the particle sizes [8]. It is well known that 5 -FU is negatively charged; since the $\mathrm{pKa}$ of the $5-\mathrm{FU}$ is 8.0 and the $\mathrm{pH}$ of $5-\mathrm{FU}$ solution is $8.4,70 \%$ of $5-\mathrm{FU}$ is ionized and negatively charged in the solution [31]. Thus, an electrostatic attraction might exist between chitosan and the negatively charged drug while ionic gelation was performed between chitosan and TPP [30]. Since 5-FU amount in chitosan solution increases, former chitosan structure may lead to simple nanocomplexes with reduced size particles and less compact and uniform structure (Table 4). The increase of PDI values of nanoparticles with the increase of 5-FU amount incorporated in chitosan solutions is clear in Table 4 . Moreover, as a visual observation, only 5-FU encapsulated chitosan nanoparticles ( $0.5 \% 5-\mathrm{FU})$ formed white opalescent solution which indicates chitosan nanoparticles formed successfully (ionic gelation that occurred) while others were all clear, which may be due to the fact that clear solutions might be in the form of nanocomplex structure other than nanoparticles. Figures 2(a) and 2(b) demonstrate average size diameters of chitosan and $0.5 \% 5$-FU encapsulated chitosan nanoparticles with narrow size distributions. As seen from Figures 2(c) and 2(d), SEM images, verifiing the nanoscale size and spherical shape of the particles, supported the

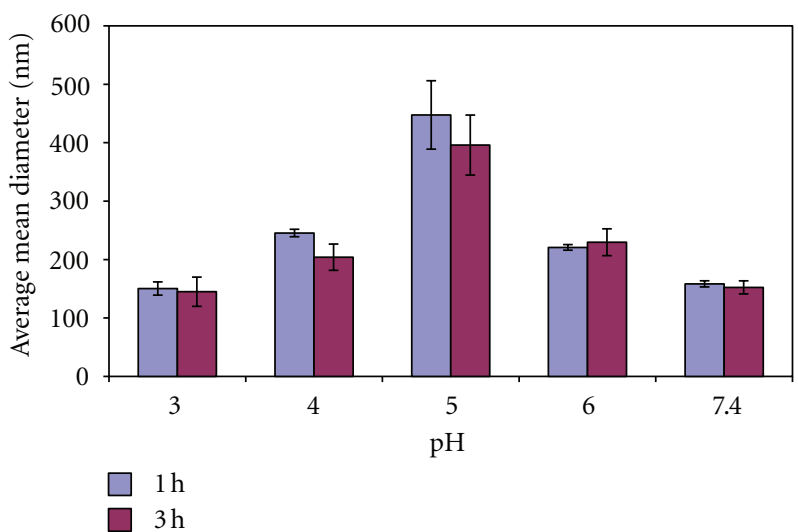

FIgURE 1: Average mean diameters of chitosan nanoparticles incubated in different $\mathrm{pH}$ values of phosphate buffer solutions after 1 and $3 \mathrm{~h}$ incubation period.

size measurement by DLS analysis with Zetasizer Nano $\mathrm{S}$ (Malvern, UK).

5-FU carrying capacity in terms of the encapsulation efficiency is calculated and demonstrated in Table 4. The initial 5-FU loading affected the encapsulation efficiency for chitosan nanoparticles, as is evident in Table 4, where the values range from 29.98 to $69.69 \%$. Previously, it was reported that 5-FU encapsulation efficiency of chitosan nanoparticles decreased by elevation of 5-FU amounts during formation of nanoparticles [8]. In this study, results are in good correlation with the reported study.

Figure 2(e) demonstrates FTIR spectra of chitosan and 5-FU encapsulated chitosan nanoparticles. For chitosan, the general characteristic absorption bands of the amide I band, $\mathrm{N}-\mathrm{H}$ bending, and C-N stretching are at $(1700-1600) \mathrm{cm}^{-1}$, (1500-1550) $\mathrm{cm}^{-1}$, and (2800-2900) $\mathrm{cm}^{-1}$, respectively. As seen from Figure 2(e), amide I band and $\mathrm{N}-\mathrm{H}$ bending highly shifted to 1570 ( $\mathrm{N}-\mathrm{H}$ stretching vibration of $\mathrm{NH}_{3}{ }^{+}$group) and $1406 \mathrm{~cm}^{-1}$, respectively, which may be due to strong ionic crosslinking of chitosan and TPP. Moreover, peaks at $1364 \mathrm{~cm}^{-1}$ (aromatic ring), $771 \mathrm{~cm}^{-1}$ (vibration of $\mathrm{CF}=\mathrm{CH}$ group), and $3000-3500 \mathrm{~cm}^{-1}$ (the presence of more $\mathrm{F}$ (unbound) groups from the 5-FU) greatly showed successful encapsulation of 5-FU into chitosan nanoparticle.

3.4. In Vitro Release of 5-FU from Chitosan Nanoparticles. In order to investigate 5-FU release behavior of chitosan nanoparticles, they were incubated in different release media 


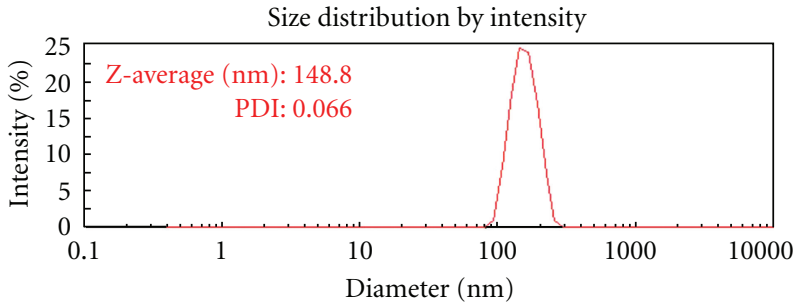

(a)

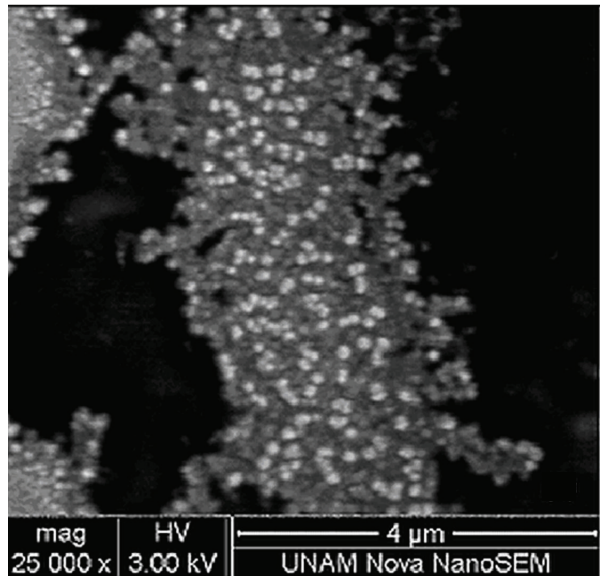

(c)

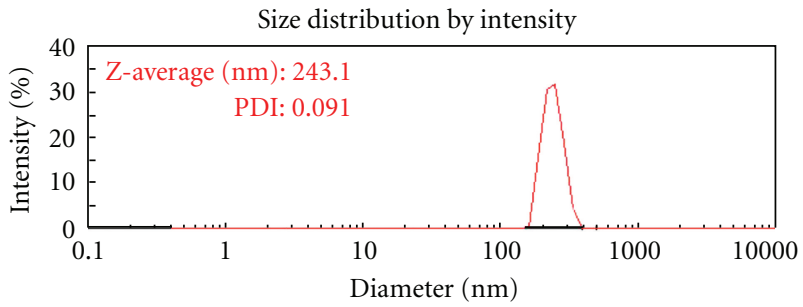

(b)

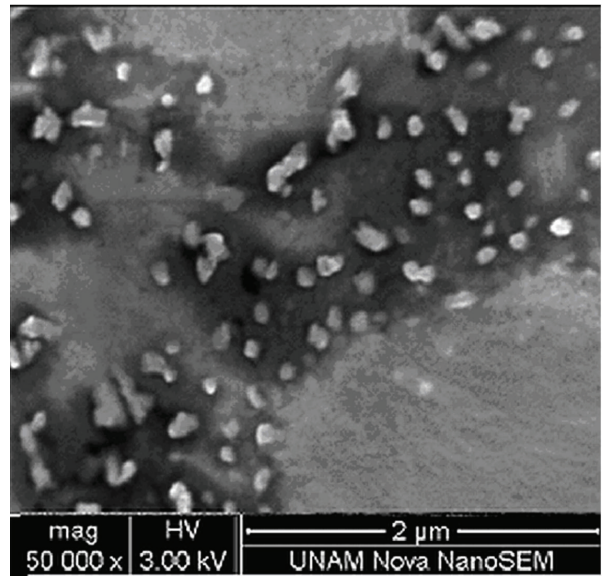

(d)

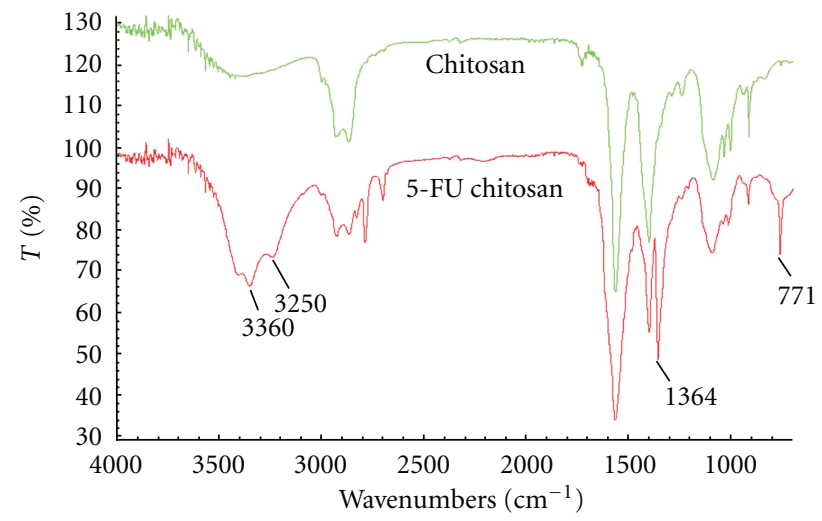

(e)

FIGURE 2: Particle size distributions of chitosan (a) and 5-FU encapsulated chitosan nanoparticles (b). SEM images of 5-FU encapsulated chitosan nanoparticles (c) 25000x (d) 50000x. FTIR spectra of chitosan and 5-FU encapsulated chitosan nanoparticles (e).

(phosphate buffer pH: 3, 4, 5, 6, and 7.4) and assessed by UV spectrophotometry. Figure 3 demonstrates 5 -FU release profiles up to $408 \mathrm{~h}$ of incubation period. As shown in Figure 3, chitosan nanoparticles showed an initial burst release of 5 -FU in a period of $6 \mathrm{~h}$ for all incubation media, which was in the range of $12.7-21.2 \%$. This initial rapid release, characterized as "burst effect", is due to the fact that some amounts of 5-FU were localized on the surface of nanoparticles by adsorption which could be released easily by diffusion. After this initial burst effect, a slower sustained and controlled release occurred throughout the incubation period and the release amount is in the range of $29.1-60.8 \%$. Release profiles supported that 5-FU molecules were encapsulated among the positively charged hydrophilic chains; in the mean time 5-FU absorption onto the external surfaces of the chitosan nanoparticles occurred.

In this study, 5-FU was used as a model drug to investigate $\mathrm{pH}$-responsive release behavior of chitosan nanoparticles, since 5-FU drug has been used in cancer therapy. The release kinetics at $\mathrm{pH} 3$ to 7.4 within $408 \mathrm{~h}$ clearly indicated that $\mathrm{pH}$ strongly influenced 5-FU release from chitosan scaffolds (Figure 3). At pH 3 and 7.4, only about $29.1 \%$ and $34.1 \%$ amounts of 5-FU were released after $408 \mathrm{~h}$, respectively. The results may be due to the fact that the nanoparticles in shrinking state entrapped drug tightly and prevent it from penetrating through the compact structure. On the other hand, a continuous and fast release profile could be found when the sample was incubated in $\mathrm{pH} 5.0$ 
TABLE 5: Mathematical models and parameters based on release data.

\begin{tabular}{|c|c|c|c|c|c|c|c|c|}
\hline & Zero-order & First-order & Higuchi’s & Hixson-Crowell's & Korsmeyer-Peppas's & & Kopcha's & \\
\hline $\mathrm{pH}$ & $\begin{array}{c}\text { Correlation } \\
\text { value }\left(R^{2}\right)\end{array}$ & $\begin{array}{c}\text { Correlation } \\
\text { value }\left(R^{2}\right)\end{array}$ & $\begin{array}{c}\text { Correlation } \\
\text { value }\left(R^{2}\right)\end{array}$ & $\begin{array}{c}\text { Correlation } \\
\text { value }\left(R^{2}\right)\end{array}$ & $\begin{array}{c}\text { Correlation } \\
\text { value }\left(R^{2}\right)\end{array}$ & $n$ & $\begin{array}{c}\text { Correlation } \\
\text { value }\left(R^{2}\right)\end{array}$ & $A / B$ \\
\hline 3.0 & 0.77 & 0.81 & 0.93 & 0.80 & 0.80 & 0.28 & 0.89 & 36.87 \\
\hline 4.0 & 0.76 & 0.82 & 0.93 & 0.80 & 0.80 & 0.30 & 0.93 & 37.12 \\
\hline 5.0 & 0.81 & 0.89 & 0.96 & 0.87 & 0.87 & 0.34 & 0.97 & 43.08 \\
\hline 6.0 & 0.76 & 0.82 & 0.93 & 0.80 & 0.80 & 0.30 & 0.92 & 36.93 \\
\hline 7.4 & 0.75 & 0.79 & 0.92 & 0.78 & 0.78 & 0.31 & 0.93 & 36.91 \\
\hline
\end{tabular}

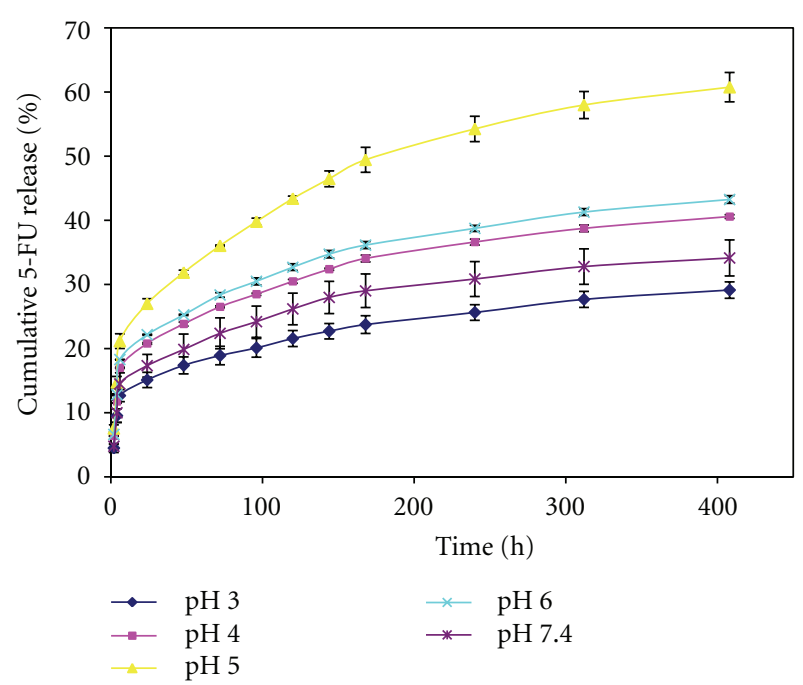

FIGURE 3: In vitro release profiles of 5-FU from chitosan nanoparti-

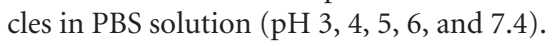

buffer. As shown in Figure 3, 60.8\% of 5-FU released after $408 \mathrm{~h}$, which may be due to the swelling behavior of chitosan nanoparticles with regard to $\mathrm{pH}$ sensitivity. Results conclude that 5-FU encapsulated chitosan nanoparticles are strongly dependent on $\mathrm{pH}$, which may be due to the stability properties of 5-FU encapsulated chitosan nanoparticles depending on the $\mathrm{pH}$ value. Drug release behaviors of 5-FU encapsulated nanoparticles suggested labile behavior of nanoparticles under different $\mathrm{pH}$ values. Thus, this $\mathrm{pH}$ sen-sitivity would be useful for drug release for the targeted tumor regions. Chitosan nanoparticles not only prevented releasing most of the drug in normal cell or tissue but also guarantied releasing efficient amount the drug in the targeted area.

3.5. Release Kinetics and Mechanisms of Drug Release. Understanding mechanism for release of drugs from nanoparticles has been well described in the literature, suggesting the mechanism to be desorption, diffusion, and matrices degradation. However, recent studies focus on biopolymers responsive to physiological changes such as $\mathrm{pH}$, temperature, and external stimuli that can trigger a control release of the therapeutic agent [32]. In this study, the release kinetics and mechanisms of 5-FU release from chitosan nanoparticles were evaluated by several mathematical models (zero order, first order, Higuchi, Hixson-Crowell, Korsmeyer-Peppas, and Kopcha). Table 5 demonstrates correlation values $\left(R^{2}\right)$ and release parameters determined from the results of model fitting of the release profiles. As seen from Table 5, according to correlation values, release data well fitted to the Higuchi model on all $\mathrm{pH}$ values evaluated which indicates that 5FU is released by diffusion. Moreover, the Korsmeyer-Peppas release model (high correlation values) exponent, $n$, is about 0.3 , which confirms that the Fickian diffusion is the controlling factor in drug release. Since the Kopcha model can easily be used to help quantify the contribution of diffusion and polymer relaxation, results shown in Table 2 greatly improve that drug release, occurred mainly as a result of the Fickian diffusion with regarding to the value of $A$, is far greater than the value of $B$.

Beside the mechanism of nanoparticles, the hydrogelbased delivery systems are of two major categories: (i) timecontrolled systems and (ii) stimuli-induced release systems [33]. Although the release mechanism is diffusion for all cases, chitosan nanoparticles incubated for different $\mathrm{pH}$ values, the Kopcha model gives the clue of $\mathrm{pH}$ sensitivity of chitosan nanoparticles due to the wide range of $A / B$ values (36.87-43.08). Further, bad fitting release data of the HixsonCrowell model suggests that there is no change in surface area as a function of time and also may confirm that the change depends on the stimuli-induced release systems.

\section{Conclusions}

In this study, chitosan nanoparticle formation was optimized in terms of localized drug delivery systems for cancer treatment studies. Production of chitosan nanoparticle was explored by varying conditions, and more significantly chitosan nanoparticles with $148.8 \pm 1.1 \mathrm{~nm}$ size were prepared, which revealed critical properties during chitosan nanoparticle formation. Furthermore, chitosan nanoparticles were demonstrated as $\mathrm{pH}$ sensitive with respect to instantaneous swelling ( $\mathrm{pH}: 3$ to 5 ) and shrinking ( $\mathrm{pH}$ : 5 to 7.4 ) responses. 5 -FU encapsulation of chitosan nanoparticles achieved as $\sim 70 \%$ with $\sim 250 \mathrm{~nm}$ diameter size, indicating potential use for drug delivery applications. In vitro release studies of 5FU encapsulated chitosan nanoparticles showed a sustained and controlled release after a minimal burst release of 5FU. Additionally, release profiles demonstrated significant 
dependence on $\mathrm{pH}$, leading chitosan nanoparticles as good candidates for use in tumor localized drug delivery. 5-FU encapsulated chitosan nanoparticles not only would offer several advantages over conventional drug therapies but also expected to overcome side effects regarding to dosing and toxicity. However, further optimization studies including stabilization and targeting should be performed both in vitro and in vivo.

\section{Acknowledgment}

The authors thank the Turkish National Research Council (TÜBİTAK) for supporting Dr. R. Seda Tığlı Aydın as a postdoctoral fellow.

\section{References}

[1] S. S. Feng, "Nanoparticles of biodegradable polymers for newconcept chemotherapy," Expert Review of Medical Devices, vol. 1, no. 1, pp. 115-125, 2004.

[2] L. Brannon-Peppas and J. O. Blanchette, "Nanoparticle and targeted systems for cancer therapy," Advanced Drug Delivery Reviews, vol. 56, no. 11, pp. 1649-1659, 2004.

[3] H. Devalapally, A. Chakilam, and M. M. Amiji, "Role of nanotechnology in pharmaceutical product development," Journal of Pharmaceutical Sciences, vol. 96, no. 10, pp. 2547-2565, 2007.

[4] W. Paul and C. P. Sharma, "Chitosan, a drug carrier for the 21st century: a review," S.T.P. Pharma Sciences, vol. 10, no. 1, pp. 5-22, 2000.

[5] J. M. Dang and K. W. Leong, "Natural polymers for gene delivery and tissue engineering," Advanced Drug Delivery Reviews, vol. 58, no. 4, pp. 487-499, 2006.

[6] K. A. Janes, P. Calvo, and M. J. Alonso, "Polysaccharide colloidal particles as delivery systems for macromolecules," Advanced Drug Delivery Reviews, vol. 47, no. 1, pp. 83-97, 2001.

[7] P. Calvo, C. Remuñan-López, J. L. Vila-Jato, and M. J. Alonso, "Chitosan and chitosan/ethylene oxide-propylene oxide block copolymer nanoparticles as novel carriers for proteins and vaccines," Pharmaceutical Research, vol. 14, no. 10, pp. 14311436, 1997.

[8] H. C. Yang and M. H. Hon, "The effect of the molecular weight of chitosan nanoparticles and its application on drug delivery," Microchemical Journal, vol. 92, no. 1, pp. 87-91, 2009.

[9] K. A. Janes, M. P. Fresneau, A. Marazuela, A. Fabra, and M. J. Alonso, "Chitosan nanoparticles as delivery systems for doxorubicin," Journal of Controlled Release, vol. 73, no. 2-3, pp. 255-267, 2001.

[10] Q. Gan and T. Wang, "Chitosan nanoparticle as protein delivery carrier-Systematic examination of fabrication conditions for efficient loading and release," Colloids and Surfaces B, vol. 59, no. 1, pp. 24-34, 2007.

[11] J. L. Zhang, R. S. Srivastava, and R. D. K. Misra, "Coreshell magnetite nanoparticles surface encapsulated with smart stimuli-responsive polymer: synthesis, characterization, and LCST of viable drug-targeting delivery system," Langmuir, vol. 23, no. 11, pp. 6342-6351, 2007.

[12] J. Cha, W. B. Lee, C. R. Park, Y. W. Cho, C. H. Ahn, and I. C. Kwon, "Preparation and characterization of cisplatinincorporated chitosan hydrogels, microparticles, and nanoparticles," Macromolecular Research, vol. 14, no. 5, pp. 573578, 2006.
[13] T. López-León, E. L. S. Carvalho, B. Seijo, J. L. Ortega-Vinuesa, and D. Bastos-González, "Physicochemical characterization of chitosan nanoparticles: electrokinetic and stability behavior," Journal of Colloid and Interface Science, vol. 283, no. 2, pp. 344351, 2005.

[14] A. Shah, W. MacDonald, and J. Goldie, "5-FU infusion in advanced colorectal cancer: a comparison of three dose schedules," Cancer Treatment Reports, vol. 69, no. 7-8, pp. 739-742, 1985.

[15] G. A. Caballero, R. K. Ausma, and E. J. Quebbema, "Longterm, ambulatory, continuous infusion of 5-FU for the treatment of advanced adenocarcinoma," Cancer Treatment Reviews, vol. 69, pp. 13-16, 1985.

[16] N. Rapoport, "Physical stimuli-responsive polymeric micelles for anti-cancer drug delivery," Progress in Polymer Science, vol. 32, no. 8-9, pp. 962-990, 2007.

[17] E. K. Lim, Y. M. Huh, J. Yang, K. Lee, J. S. Suh, and S. Haam, "PH-triggered drug-releasing magnetic nanoparticles for cancer therapy guided by molecular imaging by MRI," Advanced Materials, vol. 23, no. 21, pp. 2436-2442, 2011.

[18] T. P. Hadjiioannou, G. D. Christian, M. A. Koupparis, and P. E. Macheras, Quantitative Calculations in Pharmaceutical Practice and Research, VCH Publishers, New York, NY, USA, 1993.

[19] D. W. A. Bourne, "Pharmacokinetics," in Modern Pharmaceutics, G. S. Banker and C. T. Rhodes, Eds., pp. 67-93, Marcel Dekker, New York, NY, USA, 2002.

[20] T. Higuchi, "Mechanism of sustained-action medication. Theoretical analysis of rate of release of solid drugs dispersed in solid matrices," Journal of Pharmaceutical Sciences, vol. 52, pp. 1145-1149, 1963.

[21] A. W. Hixson and J. H. Crowell, "Dependence of reaction velocity upon surface and agitation," Industrial \& Engineering Chemistry, vol. 23, pp. 923-931, 1931.

[22] R. W. Korsmeyer, R. Gurny, and E. Doelker, "Mechanisms of solute release from porous hydrophilic polymers," International Journal of Pharmaceutics, vol. 15, no. 1, pp. 25-35, 1983.

[23] M. Kopcha, N. G. Lordi, and K. J. Tojo, "Evaluation of release from selected thermosoftening vehicles," Journal of Pharmacy and Pharmacology, vol. 43, no. 6, pp. 382-387, 1991.

[24] Q. Gan, T. Wang, C. Cochrane, and P. McCarron, "Modulation of surface charge, particle size and morphological properties of chitosan-TPP nanoparticles intended for gene delivery," Colloids and Surfaces B, vol. 44, no. 2-3, pp. 65-73, 2005.

[25] L. Qi, Z. Xu, X. Jiang, C. Hu, and X. Zou, "Preparation and antibacterial activity of chitosan nanoparticles," Carbohydrate Research, vol. 339, no. 16, pp. 2693-2700, 2004.

[26] A. M. De Campos, A. Sánchez, and M. J. Alonso, "Chitosan nanoparticles: a new vehicle for the improvement of the delivery of drugs to the ocular surface. Application to cyclosporin A," International Journal of Pharmaceutics, vol. 224, no. 1-2, pp. 159-168, 2001.

[27] F. L. Mi, S. S. Shyu, C. Y. Kuan, S. T. Lee, K. T. Lu, and S. F. Jang, "Chitosan-Polyelectrolyte complexation for the preparation of gel beads and controlled release of anticancer drug: I: effect of phosphorous polyelectrolyte complex and enzymatic hydrolysis of polymer," Journal of Applied Polymer Science, vol. 74, pp. 1868-1879, 1999.

[28] J. Zhao and J. Wu, "Preparation and characterization of the fluorescent chitosan nanoparticle probe," Chinese Journal of Analytical Chemistry, vol. 34, no. 11, pp. 1555-1559, 2006.

[29] H. Katas and H. O. Alpar, "Development and characterisation of chitosan nanoparticles for siRNA delivery," Journal of Controlled Release, vol. 115, no. 2, pp. 216-225, 2006. 
[30] N. Csaba, M. Köping-Höggård, and M. J. Alonso, "Ionically crosslinked chitosan/tripolyphosphate nanoparticles for oligonucleotide and plasmid DNA delivery," International Journal of Pharmaceutics, vol. 382, no. 1-2, pp. 205-214, 2009.

[31] M. Kondo and M. Araie, "Iontophoresis of 5-fluorouracil into the conjunctiva and sclera," Investigative Ophthalmology and Visual Science, vol. 30, no. 3, pp. 583-585, 1989.

[32] C. Gonçalves, P. Pereira, and M. Gama, "Self-assembled hydrogel nanoparticles for drug delivery applications," Materials, vol. 3, pp. 1420-1460, 2010.

[33] M. Hamidi, A. Azadi, and P. Rafiei, "Hydrogel nanoparticles in drug delivery," Advanced Drug Delivery Reviews, vol. 60, no. 15, pp. 1638-1649, 2008. 

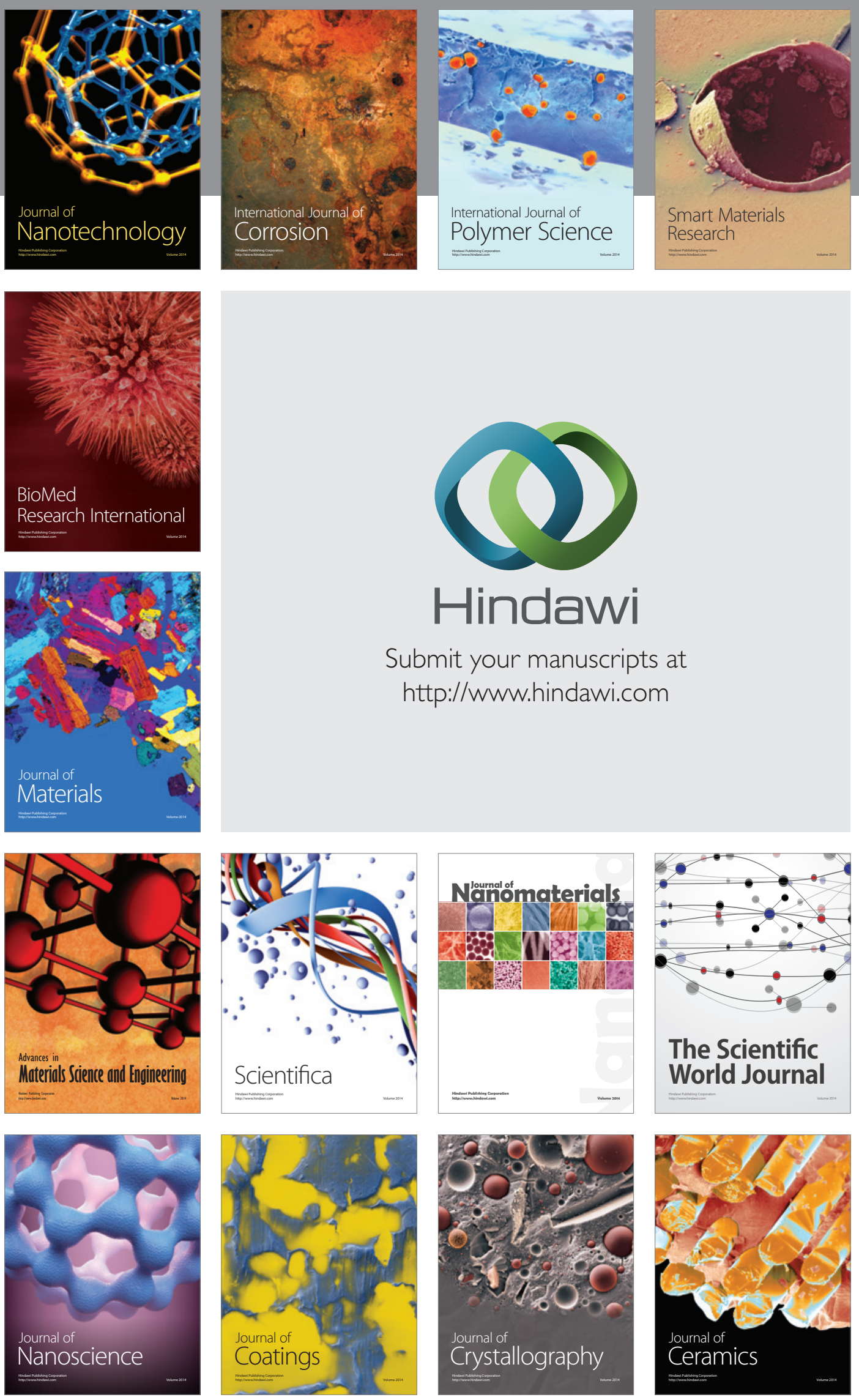

The Scientific World Journal

Submit your manuscripts at

http://www.hindawi.com

\section{World Journal}

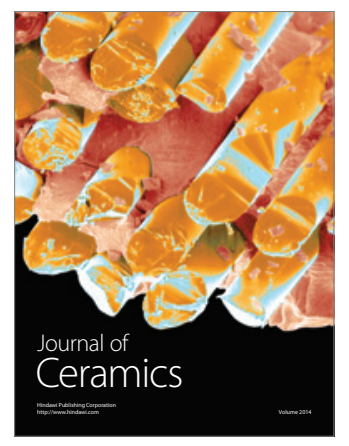

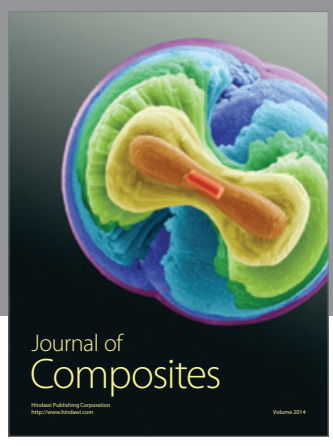
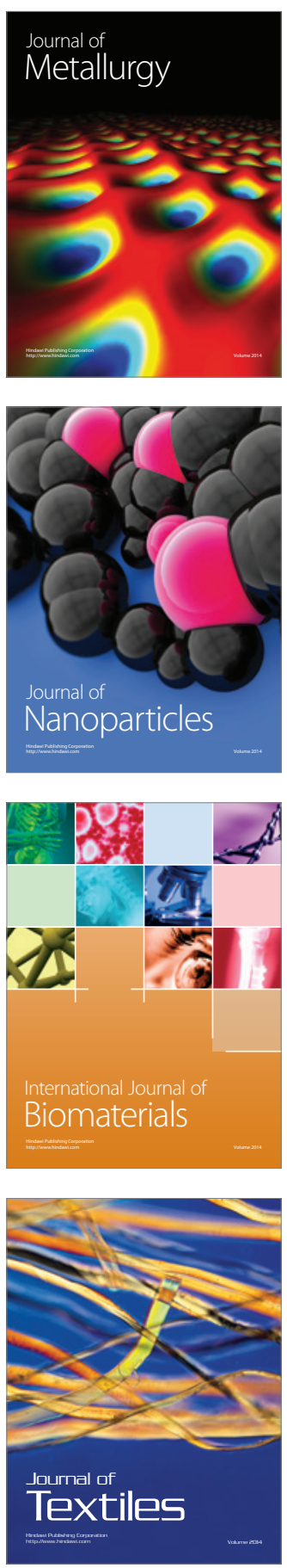